\title{
Lentinan, a Shiitake Mushroom $\beta$-Glucan, Downregulates the Enhanced PD-L1 Expression Induced by Platinum Compounds in Gastric Cancer Cells
}

\author{
Hiroko Ina ${ }^{1}$, Kenji Ina ${ }^{2, *}$, Megumi Kabeya ${ }^{3}$, Satoshi Kayukawa ${ }^{4}$, Takashi Yoshida ${ }^{4}$ and \\ Masahiko Yoneda ${ }^{1}$
}

${ }^{1}$ School of Nursing and Health, Aichi Prefectural University; ${ }^{2}$ Department of Psychosomatic Medicine; ${ }^{3}$ Department of Pharmacy; ${ }^{4}$ Department of Clinical Oncology, Nagoya, Japan

\begin{abstract}
Background: Despite recent therapeutic improvements, the prognosis of unresectable gastric cancer remains poor. Upregulation of programmed cell death ligand 1 (PD-L1) in tumor cells is believed to be an important mechanism to escape from the host's immune response. The expression of PD-L1 in tumors is regulated in a highly complex manner by various upstream signaling molecules, depending on the cell type. Given that the efficacy of chemotherapeutic agents for metastatic gastric cancer is limited due to immune escape caused by enhanced PD-L1 expression, PD-1/PD-L1 targeted immunotherapy may be a promising alterative for chemotherapy. However, immune checkpoint inhibitor monotherapy has shown clinical benefits in less than $20 \%$ of patients with gastric cancer and its underlying mechanism remains to be elucidated. On the other hand, lentinan, a $\beta$-glucan purified from Shiitake mushrooms, has significant immune-stimulating effects and has been reported to improve survival in patients with metastatic gastric cancer receiving chemotherapy. In the current study we investigated the mechanism by which lentinan increases the chemotherapeutic efficacy by focusing on the expression of PD-L1.
\end{abstract}

Methods: To evaluate the effects of lentinan as well as antineoplastic agents, the expression of PD-L1 and associated molecules was analyzed by real-time polymerase chain reaction and western blotting using the human gastric cancer cell lines, NUGC3, MKN1, and MKN45.

Results: Treatment with either cisplatin or oxaliplatin dose-dependently enhanced PD-L1 mRNA and protein expression through the mitogen-activated protein kinase (MAPK) pathway in gastric cancer cells. However, lentinan treatment inhibited the platinum drug-stimulated expression of PD-L1 in gastric cancer cells mainly by suppressing MAPK signaling without affecting the phosphatidylinositol-3 kinase/AKT pathway or transcription factors.

Conclusions: Platinum-based drugs enhanced the expression of PD-L1 via the MAPK pathway in gastric cancer cells. Lentinan downregulated PD-L1 expression induced by either cisplatin or oxaliplatin, suggesting that a combination of this $\beta$-glucan and platinum-based chemotherapy could restore the chemosensitivity of cells.

Keywords: Lentinan, gastric cancer, programmed cell death ligand 1, mitogen-activated protein kinase.

\section{BACKGROUND}

Gastric cancer remains the fifth most common malignancy and the third leading cause of cancerrelated mortality worldwide [1]. For patients with metastatic gastric cancer, a combination of platinum and fluoropyrimidine is considered the mainstay of first line of treatment [2,3]. Despite recent therapeutic improvements, the prognosis of patients with unresectable gastric cancer receiving chemotherapy is poor $[4,5]$. The efficacy of chemotherapeutic agents is severely limited due to adverse effects and resistance to conventional treatments.

Cancer cells express many inhibitory signaling proteins that enable their survival in the host. Such immune evasion is essential for cancer development, progression, and chemo-resistance [6]. One such inhibitory molecule is programmed cell death ligand 1

*Address correspondence to this author at the Department of Psychosomatic Medicine, Nagoya Memorial Hospital, 4-305 Hirabari, Tenpaku-ku, Nagoya, 468-8520, Japan; Tel: +052-804-1111; Fax: +052-803-8830;

E-mail: kina@hospy.or.jp
(PD-L1), which engages programmed cell death receptor 1 (PD-1) expressed by activated $T$ cells and subsequently triggers inhibitory signaling pathways downstream of the T-cell antigen receptors [7, 8]. Recent evidences suggest that PD-L1 protein is abundantly expressed on the cell surface in various human cancers $[9,10]$. This protein can shield tumor cells and protect them from lysis via cytotoxic $T$ lymphocytes, suggesting that upregulation of PD-L1 in cancer cells might mediate immune escape [11]. Hence, PD-1/PD-L1 targeted immunotherapy might be promising for the treatment of metastatic gastric cancer. However, monotherapy with immune checkpoint inhibitors (ICls) affords clinical benefits in less than $20 \%$ of patients with gastric cancer and factors that determine whether a tumor responds to immunotherapy or not remain to be elucidated. Furthermore, the levels of PD-L1 in tumors are regulated in a highly complex manner by several factors, which vary depending on the cell type [12]. In melanoma and lung adenocarcinoma cells, the expression of PD-L1 was reported to be increased via 
activation of the mitogen-activated protein kinase (MAPK) pathway $[13,14]$. Stimulation of the phosphatidylinositol-3 kinase (PI3K)/AKT pathway has been associated with the intrinsic induction of PD-L1 in glioma [15]. Inflammatory signaling may also regulate PD-L1 expression. The transcription factors, NF- $\kappa \beta$ and STAT3, bind to PD-L1 promoter to regulate its expression $[16,17]$. Because these molecules involved in regulation of PD-L1 expression promote cancer development by increasing cell proliferation and decreasing apoptosis, their inhibition may contribute to the enhancement of antitumor immune response. Similar to PD-L1, PD-1 interaction with PD-L2 inhibits T cell activation [18]. Previous studies have found PD-L2 to be expressed in human tumors as well as infiltrating immune cells $[19,20]$. PD-L2 status in cancer cells might be relevant to immune escape.

Lentinan, the backbone of $\beta-(1,3)$-glucan with $\beta-(1$, 6 ) branches, is an active ingredient purified from Shiitake mushrooms [21]. This $\beta$-glucan has been approved as a biological response modifier for gastric cancer treatment [22]. Lentinan has been reported to improve the overall survival of cancer patients receiving chemotherapy $[23,24]$ through its antitumor and immunomodulatory activities [25, 26], although some inconsistent results have been presented [27, 28]. Based on the findings that lentinan reduced the tumorintrinsic gene expression of PD-L1 in gastric cancer cells [29], we hypothesized that lentinan might restore sensitivity to conventional chemotherapy through downregulating PD-L1 expression induced by chemotherapeutic agents. Accordingly, we first assessed the impact of platinum and fluoropyrimidine on the expression of PD-L1 and related signaling molecules. Next, we examined the mechanism by which lentinan modulates the effects of antineoplastic agents.

\section{METHODS}

\section{Reagents and Cell Culture}

Cisplatin, lentinan, and 5-FU were purchased from Nippon Kayaku Co., Ltd. (Tokyo, Japan), Ajinomoto Co., Ltd. (Tokyo, Japan), and Kyowa Kirin Co., Ltd. (Tokyo, Japan), respectively. Oxaliplatin was kindly provided by Yakult Pharmaceutical Industry Co., Ltd. (Tokyo, Japan). Stock solutions of these agents were prepared in sterile distilled water and dissolved in culture medium immediately before use. The human gastric cancer cell lines (NUGC3, MKN1, and MKN45) were kindly provided by the Department of
Gastroenterological Surgery, Nagoya University Graduate School of Medicine (Professor Yasuhiro Kodera) and cultured in Dulbecco's modified Eagle's medium supplemented with $10 \%$ fetal bovine serum (Life Technologies Corp., Carlsbad, CA, USA), 100 $\mathrm{U} / \mathrm{mL}$ penicillin, and $100 \mu \mathrm{g} / \mathrm{mL}$ streptomycin (Life Technologies Corp.). The cells were maintained at $37^{\circ} \mathrm{C}$ in a humidified incubator with an atmosphere containing $5 \% \mathrm{CO}_{2}$. Cells were exposed to various concentrations of either antineoplastic agents or lentinan.

\section{RNA Isolation and Reverse Transcription- Polymerase Chain Reaction (RT-PCR)}

Cells were seeded in 6-well plates at a density of 5 $\times 10^{5}$ cells per well in $1 \mathrm{~mL}$ of culture medium. Two days later, cells were incubated with the indicated concentrations of lentinan overnight and then cultured with each of the three antineoplastic agents for the indicated times. Total RNA was extracted from gastric cancer cells using SV total RNA isolation system (Promega Inc., Tokyo, Japan) and RNA concentration was quantified using a spectrophotometer (GeneQuant Pro; GE Healthcare UK Ltd., Buckinghamshire, UK). Complementary DNA (cDNA) was synthesized from 1 $\mu \mathrm{g}$ of total RNA with PrimeScript ${ }^{\mathrm{TM}}$ RT Master Mix (Takara Bio Inc., Tokyo, Japan). Real-time PCR analysis was performed using a double-strand DNAspecific dye on the Thermal Cycler Dice Real Time System (version 4.02, Code TP900/TP960; Takara Bio Inc.). The reaction mixture $(20 \mu \mathrm{L})$ included: cDNA (1 $\mu \mathrm{L})$, primer $\left(1 \mu \mathrm{L}\right.$ each), $\mathrm{ddH}_{2} \mathrm{O}(9.5 \mu \mathrm{L})$, SYBR premix EX Taqll (12.5 $\mu \mathrm{L})$ (Takara Bio Inc.). The PCR amplification conditions were as follows: $95^{\circ} \mathrm{C}$ for $5 \mathrm{~min}$, followed by 45 cycles of $95^{\circ} \mathrm{C}$ for $15 \mathrm{~s}, 60^{\circ} \mathrm{C}$ for $1 \mathrm{~s}$, and then $95^{\circ} \mathrm{C}$ for $15 \mathrm{~s}, 60^{\circ} \mathrm{C}$ for $30 \mathrm{~s}$, and $95^{\circ} \mathrm{C}$ for $15 \mathrm{~s}$. The cycle threshold $(\mathrm{Ct})$ is defined as the number of cycles required for the fluorescent signal to cross the threshold. To quantify gene expression, the $\Delta \Delta \mathrm{Ct}$ method was used to compare the expression of target genes among different samples [30]. Based on this method, real-time PCR data were expressed relative to the fluorescence intensity of $\beta$-actin (housekeeping gene) in the same samples. The primer pairs used for the cDNA amplification are listed in Table 1. At least three independent experiments were performed to determine the mean and standard error (SE) values.

\section{Protein Extraction and Western Blotting}

Samples were prepared from gastric cancer cell lines according to previously reported procedures [31, 
Table 1: Primer Pairs Used for Real-Time PCR Analysis

\begin{tabular}{|c|c|c|}
\hline & Forward primer & Reverse primer \\
\hline \hline$P D-L 1$ & GGACAAGCAGTGACCATCAAG & CCCAGAATTACCAAGTGAGTCCT \\
\hline$P D-L 2$ & ATCCAACTTGGCTGCTTCAC & CTCCCAAGACCACAGGTTCA \\
\hline MAPK & CGTTGGTACAGGGCTCCAGAA & CTGCCAGAATGCAGCCTACAGA \\
\hline AKT & AGCGACGTGGCTTTGTGAA & CTAATCCATGAGGTACTGGCCAAAG \\
\hline PI3KCA & ATTTGCTCTGTTAAAGGCCGAAAG & CAGAGCTGCTTGGCGGATTAG \\
\hline NF- $K \beta$ & ACGAATGACAGAGGCGTGTATAAGG & GGGACCTTTAGACACGCAAGGA \\
\hline STAT3 & TGCCTTATCAGGGCTGGGATAC & TGCAAGTGTTGTGGCTCGAAATA \\
\hline Rasa1 & GAACACTACTGGCCAGCATCCTA & AGGCTGTGCCAAGTTGGTTAATTC \\
\hline Rasa2 & CATGGTATGATCACAGGGACCAAG & CTCCTTAATGTCACGCACGAT \\
\hline$\beta$-actin & CATGTACGTTGCTATCCAGGC & \\
\hline
\end{tabular}

32]. Cells were lysed in lysis buffer, and the lysate was incubated on ice for 20 min and centrifuged at 15,184 $x$ $\mathrm{g}$ for $10 \mathrm{~min}$ at $4{ }^{\circ} \mathrm{C}$. The supernatant was collected for protein detection, and the total protein concentration was evaluated by the bicinchoninic acid (BCA) method. Protein $(20 \mu \mathrm{g})$ from each sample was separated by sodium dodecyl sulfate-polyacrylamide gel electrophoresis using a $5 \%$ stacking gel, and then transferred onto reinforced PVDF membranes (Millipore, Bedford, MA, USA). After blocking the nonspecific sites, each membrane was probed overnight at $4^{\circ} \mathrm{C}$ with one of the following primary antibodies: PD-L1 (Abnova Corp, Taipei, Taiwan), extracellular signal-regulated kinase (ERK) $1 / 2$ (Abnova Corp), pERK1/2 (Abnova Corp), and $\beta$-actin (Cell Signaling Technology). The membranes were washed and incubated for $30 \mathrm{~min}$ at room temperature with horseradish peroxidase-conjugated anti-mouse secondary antibodies (Cell Signaling Technology). An antibody against $\beta$-actin was used to confirm equal loading and transfer of each protein from total cellular extracts. Western blots were digitalized using the GS700 Imaging Densitometer (Bio-Rad, Hercules, CA, USA), and processed with Corel Photo Paint 7.0 to adjust image brightness and contrast. The band densities were evaluated using the Molecular Analyst Software (Bio-Rad), and normalized to pertinent controls.

\section{Statistical Analysis}

Experimental data sets were analyzed by one-way analysis of variance (ANOVA) followed by Dunnett's multiple comparison test to compare cultures exposed to various concentrations of each drug. Tukey's test was applied for the comparison of multiple different cultures. Differences were considered statistically significant when $P$ values were less than 0.05 . All statistical analyses were performed with EZR (Saitama Medical Center, Jichi Medical University; http://www.jichi.ac.jp/saitama-sct/ Saitama HP.files/ statmedEN.html), which is a graphical user interface for $\mathrm{R}$ (The R Foundation for Statistical Computing, Vienna, Austria) [33]. More precisely, it is a modified version of $\mathrm{R}$ commander designed to add statistical functions frequently used in biostatistics.

\section{RESULTS}

Real-time PCR was performed to examine how chemotherapeutic agents modulate PD-L1 expression. Constitutive levels of $P D-L 1$ expression were different among the gastric cancer cell lines. The mRNA level of $P D-L 1$ was increased in gastric cancer cell lines treated with anti-cancer agents (Figures 1 and 2). $P D-L 1$ upregulation induced by treatment with either cisplatin or oxaliplatin was dose-dependent, and the changes induced by 5 -FU were not significant. In contrast, the expression of $P D-L 2$ remained unaffected by cisplatin treatment (Figure 1). The expression of upstream signaling molecules was examined to investigate the mechanism by which chemotherapeutic agents modulate PD-L1 expression. Results showed that treatment with platinum compounds increased the mRNA level of MAPK in a dose-dependent manner (Figure 3), similar to that of $P D-L 1$. However, the mRNA expression of $A K T / P I 3 K C A$ and the transcription factors, $N F-\kappa \beta$ and STAT3, showed no significant correlation with cisplatin dosages.

Pretreatment with lentinan significantly reduced the increase in $P D-L 1$ expression induced by cisplatin 


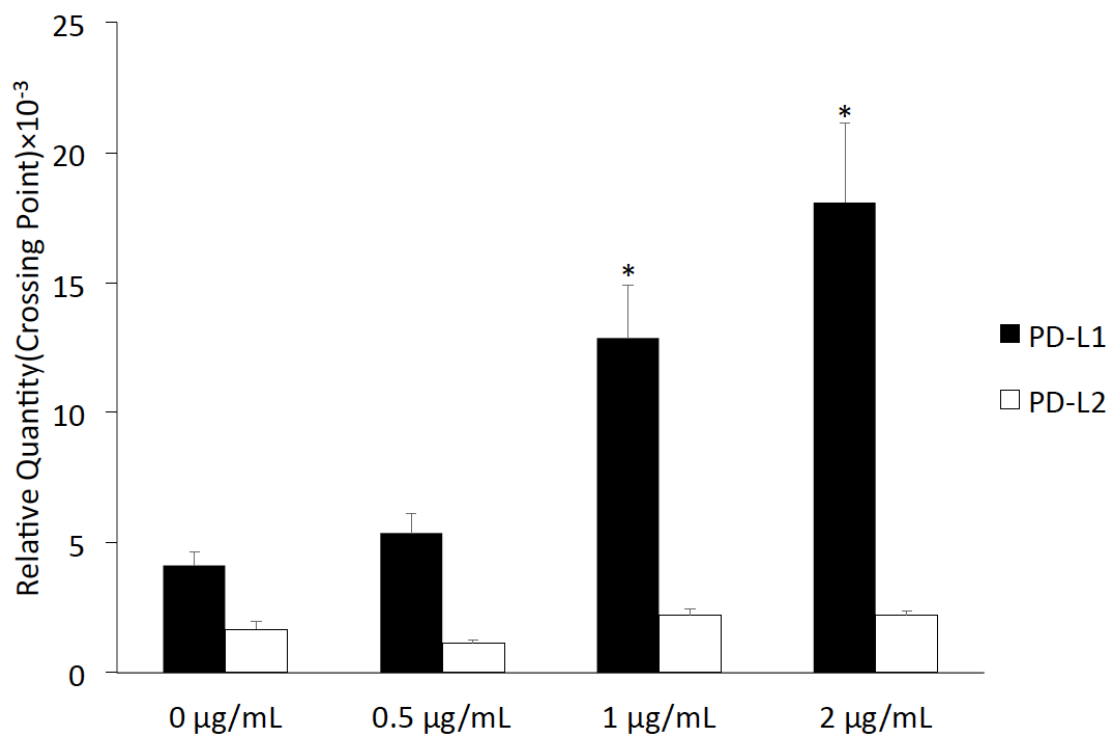

Figure 1: Effects of cisplatin on the expression of $P D-L 1$ and $P D-L 2$ in NUGC3 cells. NUGC3 cells were seeded at a density of 5 $\times 10^{5}$ cells per well in $1 \mathrm{~mL}$ of culture medium and cultured for $48 \mathrm{~h}$. Semi-confluent cultures were treated with the indicated concentrations of cisplatin for another $48 \mathrm{~h}$. Relative mRNA levels of $P D-L 1$ and $P D-L 2$ were determined by real-time PCR. Data were analyzed by the $\Delta \Delta$ Ct method [30] and normalized to $\beta$-actin, a house keeping gene. More than three independent experiments were performed to determine the mean and standard error (SE) values. Asterisk $\left({ }^{*}\right)$ indicates significant difference compared to the control group; $P<0.05$ (Dunnett's multiple comparison test).

A.

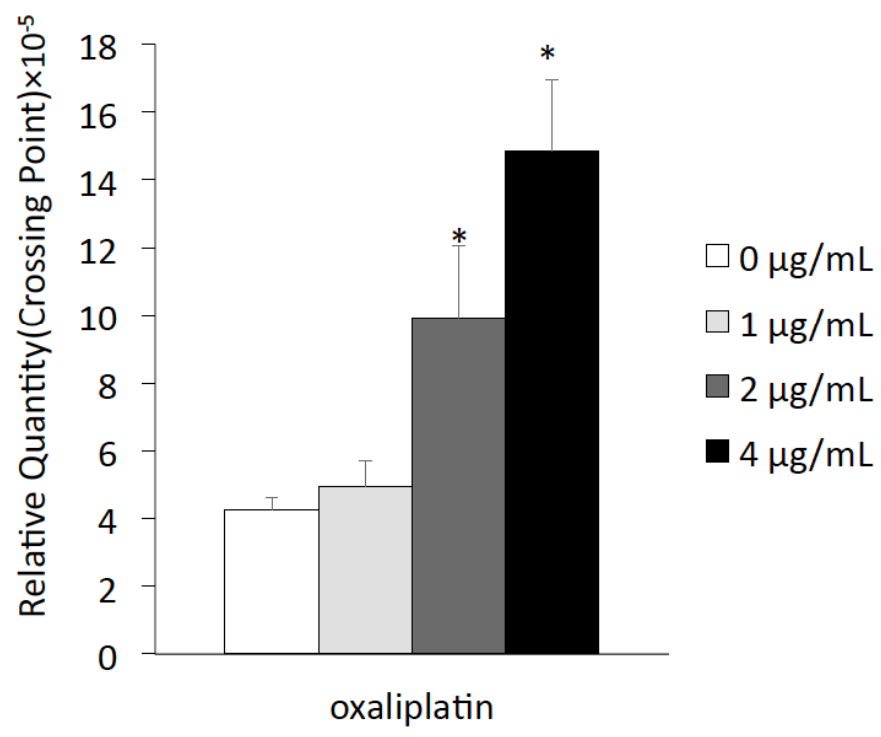

B.

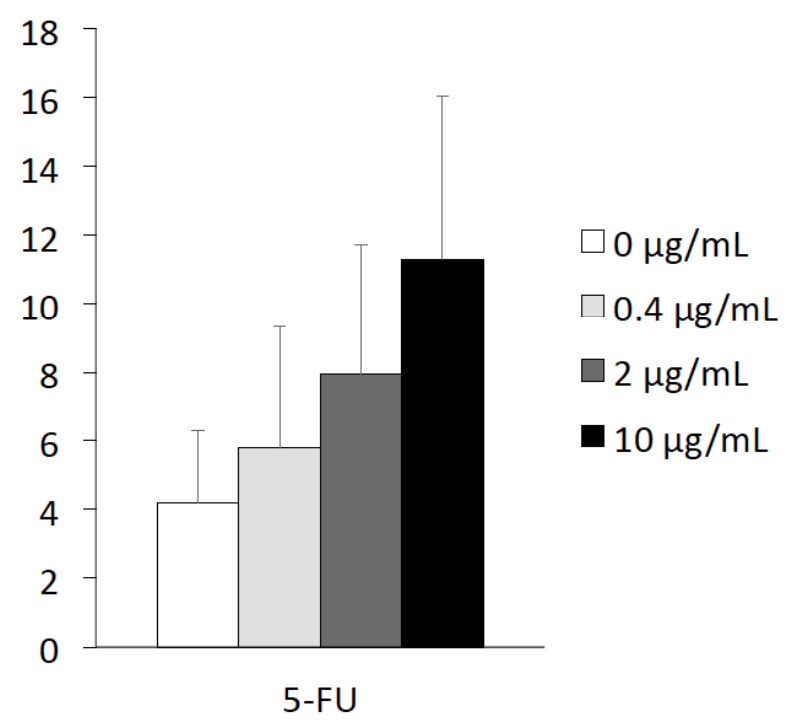

Figure 2: Effects of anticancer agents on the expression of $P D-L 1$ in MKN1 cells.

A. Oxaliplatin

B. 5-FU

MKN1 cells were seeded at a density of $5 \times 10^{5}$ cells per well in $1 \mathrm{~mL}$ of culture medium and cultured for $48 \mathrm{~h}$. Semi-confluent cultures were treated with the indicated concentrations of oxaliplatin or 5-FU for another $48 \mathrm{~h}$. Relative mRNA levels of $P D-L 1$ were determined by real-time PCR. Data were analyzed by the $\Delta \Delta$ Ct method [30] and normalized to $\beta$-actin, a house keeping gene. More than three independent experiments were performed to determine the mean and standard error (SE) values. Asterisk $\left({ }^{*}\right)$ indicates significant difference compared to the control group; $P<0.05$ (Dunnett's multiple comparison test). 
A.

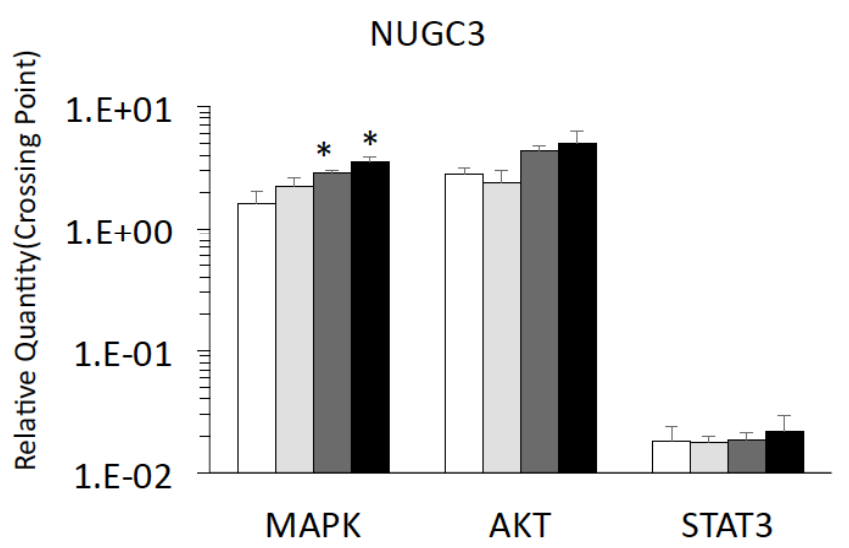

B.

\section{MKN1}

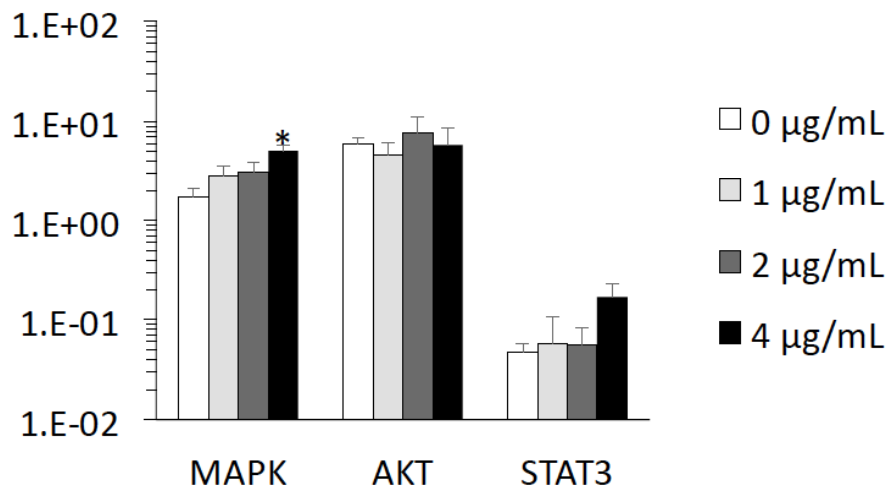

Figure 3: Effects of cisplatin on the expression of signaling molecules that regulate $P D-L 1$ expression in gastric cancer cells.

\section{A. NUGC3 cells \\ B. MKN1 cells}

Gastric cancer cells were seeded at a density of $5 \times 10^{5}$ cells per well in $1 \mathrm{~mL}$ of culture medium and cultured for $48 \mathrm{~h}$. Semiconfluent cultures were treated with the indicated concentrations of cisplatin for another $48 \mathrm{~h}$. Relative gene expression levels were determined by real-time PCR. Data were analyzed by the $\Delta \Delta \mathrm{Ct}$ method[30] and normalized to $\beta$-actin, a house keeping gene. More than three independent experiments were performed to determine the mean and standard error (SE) values. Asterisk $\left(^{*}\right)$ indicates significant difference compared to the control group; $P<0.05$ (Dunnett's multiple comparison test).

(Figure 4) at concentrations $(1 \mathrm{ng} / \mathrm{mL})$ compatible with the serum concentrations of clinical usage [29]. As for the signaling molecules, cisplatin-induced upregulation of MAPK expression was significantly inhibited by lentinan treatment, while the expression of $A K T$ and PI3KCA was not significantly affected (Figure 5). In cultures exposed to oxaliplatin, the same tendency was observed. On the contrary, treatment with 5-FU significantly increased the mRNA expression of $M A P K$, $A K T, N F-\kappa \beta$, and STAT3, but not PI3KCA, Rasa1, or Rasa2 (Table 2).

Because treatment with platinum compounds and lentinan significantly affected the mRNA expression of $P D-L 1$ and MAPK, we next evaluated their effects on protein expression. A time-course study demonstrated

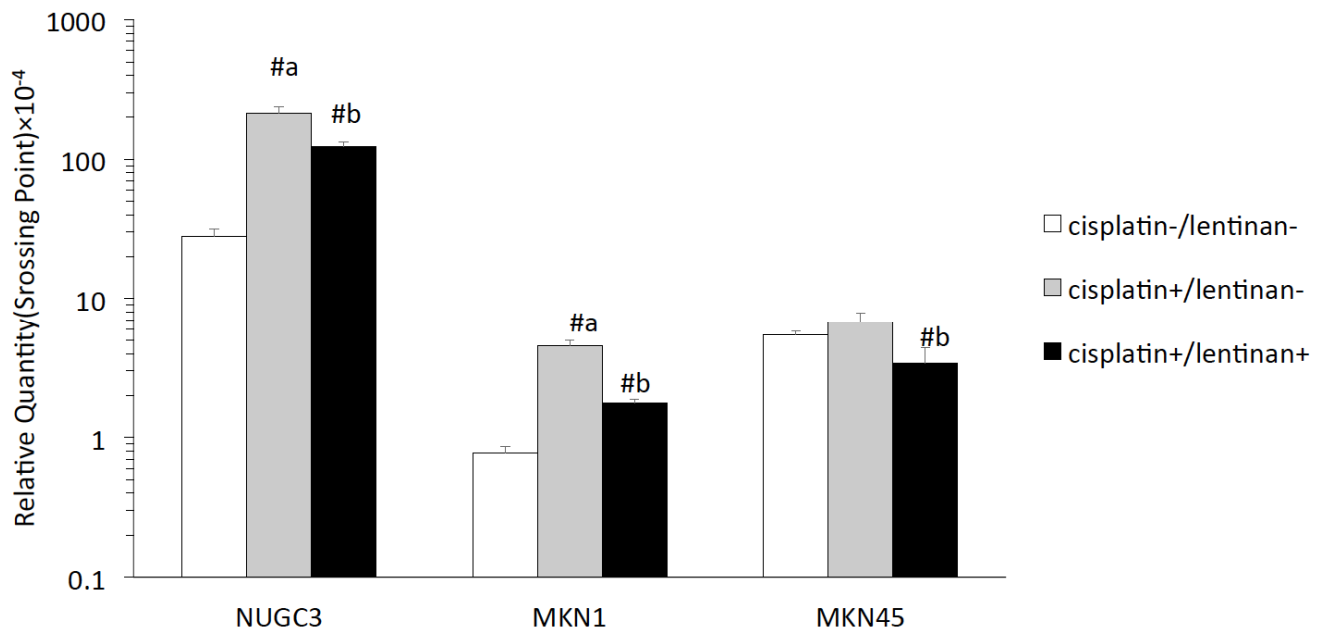

Figure 4: Lentinan significantly suppressed cisplatin-induced $P D-L 1$ expression in gastric cancer cells.

Gastric cancer cells (NUGC3, MKN1, and MKN45) were seeded at a density of $5 \times 10^{5}$ cells per well in $1 \mathrm{~mL}$ of culture medium and cultured for $48 \mathrm{~h}$. Semi-confluent cultures were pre-treated with $1 \mathrm{ng} / \mathrm{mL}$ of lentinan for $24 \mathrm{~h}$ and then treated with $1 \mu \mathrm{gg} / \mathrm{mL}$ of cisplatin for another $48 \mathrm{~h}$. Relative mRNA levels of $P D-L 1$ were determined by real-time PCR. Data were analyzed by the $\Delta \Delta \mathrm{Ct}$ method[30] and normalized to $\beta$-actin, a house keeping gene. More than three independent experiments were performed to determine the mean and standard error (SE) values. \#a indicates significant difference compared to the control cultures and $\# \mathrm{~b}$ indicates significant difference compared to the cisplatin-treated cultures; $P<0.05$ (Tukey's test). 


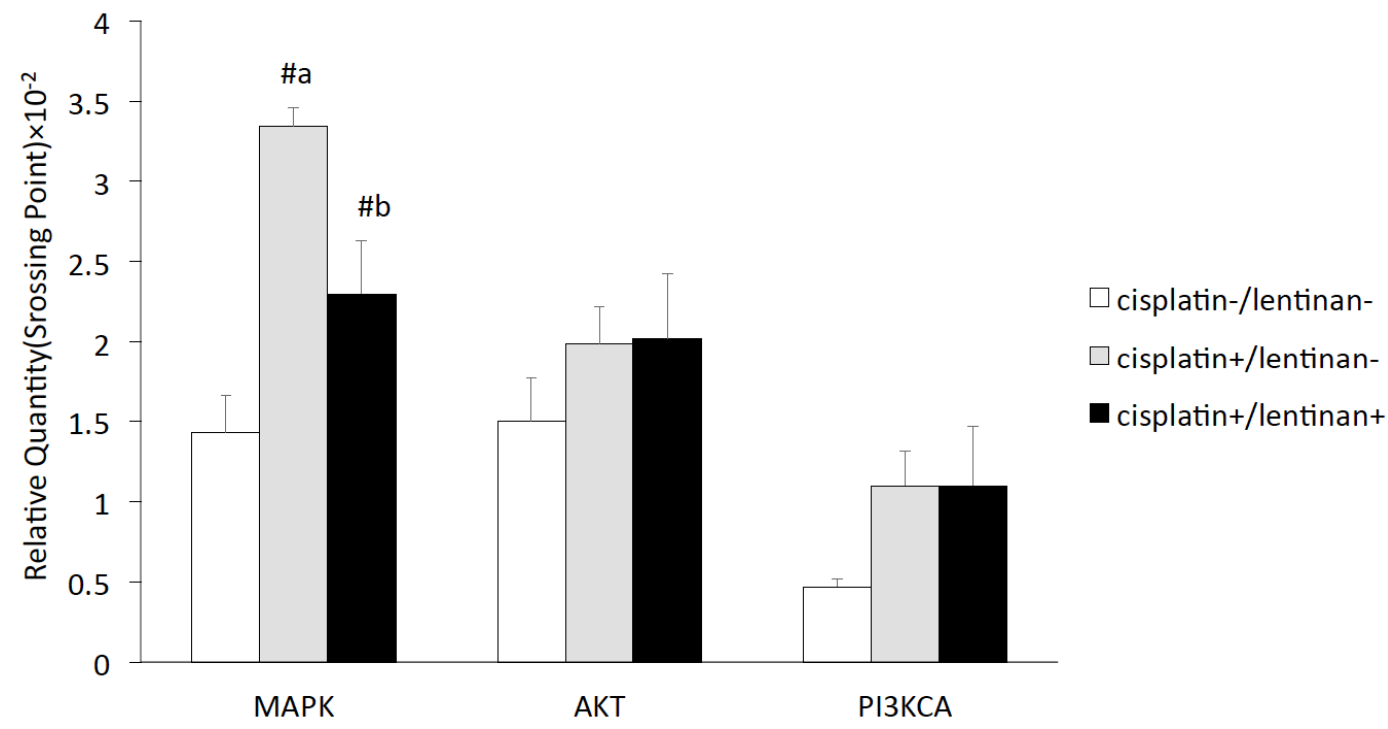

Figure 5: Effects of lentinan on the expression of signaling molecules in NUGC3 cells.

NUGC3 cells were seeded at a density of $5 \times 10^{5}$ cells per well in $1 \mathrm{~mL}$ of culture medium and cultured for $48 \mathrm{~h}$. Semi-confluent cultures were pre-treated with $1 \mathrm{ng} / \mathrm{mL}$ of lentinan for $24 \mathrm{~h}$ and then treated with $1 \mu \mathrm{g} / \mathrm{mL}$ of cisplatin for another $48 \mathrm{~h}$. Lentinan significantly suppressed cisplatin-induced expression of MAPK. However, AKT/ PI3KCA expression had no correlation with lentinan treatment. Relative mRNA levels were determined by real-time PCR. Data were analyzed by the $\Delta \Delta \mathrm{Ct}$ method [30] and normalized to $\beta$-actin, a house keeping gene. More than three independent experiments were performed to determine the mean and standard error (SE) values. \#a indicates significant difference compared to the control cultures and \#b indicates significant difference compared to the cisplatin-treated cultures $(1 \mu \mathrm{g} / \mathrm{mL}) ; P<0.05$ (Tukey's test).

Table 2: Gene Expression of Signaling Molecules Regulating PD-L1 Expression in NUGC3 Cells after 5-FU Treatment as Determined by Real-Time PCR Analysis

\begin{tabular}{|c|c|c|c|c|}
\hline 5-FU $(\boldsymbol{\mu g} / \mathbf{m L})$ & $\mathbf{0}$ & $\mathbf{0 . 4}$ & $\mathbf{2}$ & $\mathbf{1 0}$ \\
\hline \hline$M A P K\left(\times 10^{-2}\right)$ & $1.49 \pm 0.16$ & $1.90 \pm 0.16$ & $2.58 \pm 0.54^{*}$ & $3.53 \pm 0.35^{*}$ \\
\hline$A K T\left(\times 10^{-2}\right)$ & $1.62 \pm 0.14$ & $1.56 \pm 0.13$ & $2.85 \pm 0.40^{*}$ & $3.34 \pm 0.06$ \\
\hline$P I 3 K C A\left(\times 10^{-2}\right)$ & $2.13 \pm 0.88$ & $0.79 \pm 0.16$ & $1.93 \pm 0.42$ & $9.05 \pm 1.49^{*}$ \\
\hline$N F-\kappa \beta\left(\times 10^{-3}\right)$ & $4.42 \pm 0.31$ & $6.49 \pm 0.54$ & $8.41 \pm 1.33^{*}$ & $1.88 \pm 0.50$ \\
\hline STAT3 $\left(\times 10^{-5}\right)$ & $1.42 \pm 0.83$ & $2.16 \pm 0.58$ & $4.16 \pm 0.72^{*}$ & $4.66 \pm 0.32$ \\
\hline Rasa1 $\left(\times 10^{-3}\right)$ & $6.69 \pm 0.50$ & $6.25 \pm 1.24$ & $3.34 \pm 1.42$ & $3.63 \pm 0.88$ \\
\hline Rasa2 $\left(\times 10^{-3}\right)$ & $3.19 \pm 0.30$ & $3.48 \pm 0.23$ & $4.48 \pm 0.36$ \\
\hline
\end{tabular}

NUGC3 cells were seeded at a density of $5 \times 10^{5}$ cells per well in $1 \mathrm{~mL}$ of culture medium and cultured for $48 \mathrm{~h}$. Semi-confluent cultures were treated with the indicated concentrations of 5 -FU for another $48 \mathrm{~h}$. Relative levels of gene expression were determined by real-time PCR. Data were analyzed by the $\Delta \Delta \mathrm{Ct}$ method [30] and normalized to $\beta$-actin, a house keeping gene. More than three independent experiments were performed to determine the mean and standard error (SE) values. Asterisk $\left(^{*}\right)$ indicates significant difference compared to the control group; $P<0.05$ (Dunnett's multiple comparison test).

that the expression of $\mathrm{pERK} 1 / 2$ reached a peak at 30 min after stimulation with platinum compounds, while that of PD-L1 gradually increased (data not shown). Moreover, treatment with platinum compounds upregulated the protein expression of PD-L1 in a dosedependent manner. Western blot analysis revealed that pretreatment with lentinan suppressed the enhanced expression of PD-L1 and pERK1/2 induced by either cisplatin or oxaliplatin treatment in NUGC3 cells (Figure 6).

\section{DISCUSSION}

In the present study, we demonstrated that treatment with antineoplastic agents increased the level of PD-L1 expression in gastric cancer cell lines. Among these agents, treatment with cisplatin and oxaliplatin upregulated the expression of $P D-L 1$, but not $P D-L 2$, in a dose-dependent manner, implicating that PD-L1 is a platinum-inducible ligand. Exposure to 5-FU also increased the expression of $P D-L 1$; however, its dosedependency was not clear. Consistent with our 


\section{$-/-\quad C i s /-\quad$ Cis/L $\quad$ Ox/- $\quad$ Ox/L}
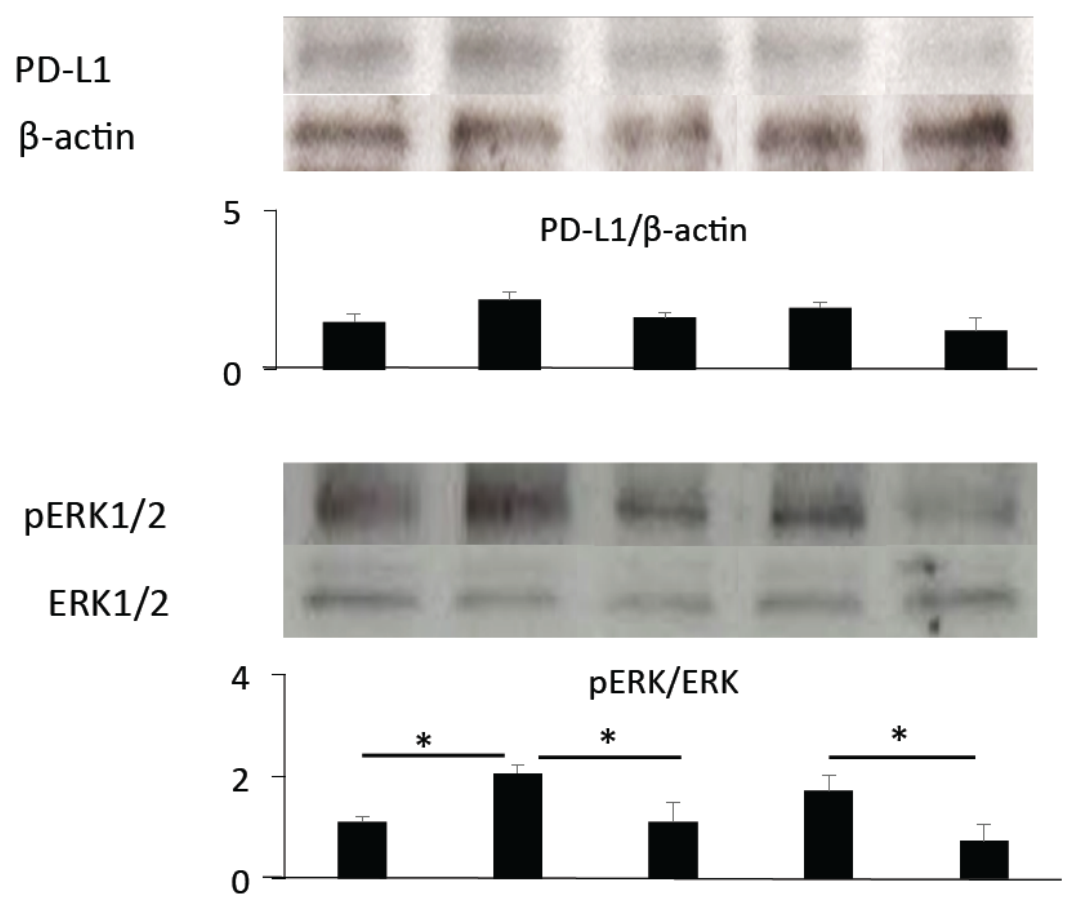

\begin{tabular}{|c|c|c|c|c|c|}
\hline & $-/-$ & Cis/ & Cis/L & Ox/ & Ox/L \\
\hline \hline cisplatin & 0 & $1 \mu \mathrm{g} / \mathrm{mL}$ & $1 \mu \mathrm{g} / \mathrm{mL}$ & 0 & $2 \mu \mathrm{g} / \mathrm{mL}$ \\
\hline oxaliplatin & 0 & 0 & 0 & $1 \mathrm{ng} / \mathrm{mL}$ & 0 \\
\hline lentinan & 0 & 0 & $2 \mu \mathrm{g} / \mathrm{mL}$ & $1 \mathrm{ng} / \mathrm{mL}$ \\
\hline
\end{tabular}

Figure 6: The protein expression of PD-L1 and pERK1/2 was analyzed by western blotting. NUGC3 cells were pretreated with 1 $\mathrm{ng} / \mathrm{mL}$ of lentinan for $24 \mathrm{~h}$ and then incubated with either $1 \mu \mathrm{g} / \mathrm{mL}$ of cisplatin or $2 \mu \mathrm{g} / \mathrm{mL}$ oxaliplatin. The incubation time for PDL1 and pERK1/2 protein was $24 \mathrm{~h}$ and $30 \mathrm{~min}$, respectively. Data are from one representative experiment among three independent experiments with similar results. Western blots were digitalized using the GS-700 Imaging Densitometer (Bio-Rad, Hercules, CA, USA), and processed with Corel Photo Paint 7.0 to adjust image brightness and contrast. The band densities were evaluated using the Molecular Analyst Software (Bio-Rad), and normalized to pertinent controls. Asterisk ( $\left.{ }^{*}\right)$ indicates significant differences among various treatment groups; $P<0.05$ (Tukey's test).

experimental findings, previous clinical studies demonstrated that platinum-based chemotherapy induced PD-L1 expression in various cancers [34, 35], which attenuated anti-tumor responses. Therefore, PDL1/PD-1 targeted immunotherapy combined with cisplatin-based chemotherapy might be a promising strategy to restore chemosensitivity [36, 37]. Pembrolizumab, an anti-PD-1 monoclonal antibody, has shown efficacy when administered as monotherapy in patients with PD-L1 tumor proportion score (TPS) > $50 \%$ [38]. The combination of platinum-based chemotherapy and pembrolizumab improved survival in patients with non-small-cell lung cancer regardless of tumor PD-L1 expression [37]. Given that the expression of PD-L1 can be upregulated by platinumbased chemotherapy even in patients with low PD-L1 TPS, the survival benefits of chemo-immunotherapy using ICls can be demonstrated across all categories of PD-L1 TPS.

Since the expression of PD-L1 is regulated by multiple signaling pathways [39, 40], optimizing such a combination of anticancer drugs and immunemodulators requires an understanding of the underlying molecular mechanisms depending on the cell type. Here, we examined the impacts of chemotherapeutic agents on signaling molecules involved in the regulation of PD-L1 expression using human gastric cancer cell lines. A series of RT-PCR experiments implicated that both cisplatin and oxaliplatin treatments increased the mRNA level of MAPK in a dosedependent manner, but had no significant effect on the expression of $A K T / P / 3 K C A . \quad N F-\kappa \beta$ is a key transcription factor involved in inflammation and is well 
known to play an essential role in interferon gammainduced $P D-L 1$ expression [41]. In the present study, our results demonstrated that $N F-\kappa \beta$ and STAT3 do not play an active role in platinum compound-induced PDL1 upregulation. On the other hand, the mechanism of PD-L1 modulation by 5-FU was more complex than that by platinum compounds; the expression of $A K T$, $N F-\kappa \beta$, STAT3, and MAPK was increased by exposure to 5-FU, but the levels of PI3KCA, Rasa1, and Rasa2 were not altered. Further studies are needed to evaluate the mechanism by which $5-F U$ affects PD-L1 expression in gastric cancer.

$\beta$-glucans are well-established natural immunemodulators with significant anti-cancer properties [26, 42], although there are remarkable differences in activities among individual glucans. Lentinan, a $\beta$ glucan purified from Shiitake mushrooms, is especially remarkable for its immunomodulating [43] and anticancer activities [44]. Additional treatment with lentinan has been reported to prolong the survival in patients with cancer, as compared to chemotherapy alone [23, 24]. Recently, it was reported that a patient showed complete disappearance of primary gastric tumor and multiple liver metastases in response to platinum-based chemotherapy combined with lentinan treatment [45]. In order to elucidate the mechanism by which lentinan enhances chemotherapeutic effects, we performed in vitro experiments. We previously demonstrated that lentinan treatment reduced the intrinsic $P D-L 1$ expression in gastric cancer cells [29]. Based on these preliminary findings, the modulatory effects of lentinan combined with antineoplastic agents were examined at the transcriptional and protein levels. Results showed that treatment with lentinan significantly inhibited cisplatin or oxaliplatin-induced $P D-L 1$ expression. This inhibitory effect was speculated to be mediated mainly via MAPK signaling, because the expression of $P D-L 1$ and MAPK was similarly decreased in the presence of lentinan. Western blot analysis demonstrated that lentinan treatment suppressed the platinum compound-induced increase in PD-L1 and pERK1/2 protein expression, which is consistent with the results of RT-PCR. Hence, lentinan may exert chemosensitizing effects through downregulating $P D-L 1$ expression.

A combination of lentinan and $\mathrm{ICls}$ may be used to enhance chemotherapeutic efficacy via regulating the PD-L1/PD-1 axis. Further investigations are necessary to establish a basis for the combinational regimens of cytotoxic chemotherapeutic agents, ICls, and lentinan for the treatment of gastric cancer.

\section{CONCLUSIONS}

In summary, our findings revealed that lentinan can inhibit cisplatin or oxaliplatin-induced increase in PD-L1 and MAPK expression, which may contribute to tumor clearance by $\mathrm{T}$-cell mediated immune responses.

\section{DECLARATIONS}

Availability of data and material: Technical appendix and dataset are available from the corresponding author at kina@hospy.or.jp

\section{COMPETING INTERESTS}

The authors have no conflict of interest to declare.

\section{AUTHOR'S CONTRIBUTIONS}

H.I. and K.I. are responsible for manuscript publication. H.I., M.Y. and K.I. designed the study and collected data. H.I., T.Y. and K.I. wrote the manuscript. M.K. and S.K. performed the statistical analysis. H.I. and M.Y. performed the in vitro experiments.

\section{REFERENCES}

[1] International Agency for Research on Cancer. GLOBOCAN 2018: estimated cancer incidence, mortality and prevalence worldwide. Available online: http://globocan.iarc.fr/ Default.aspx (accessed on 17 June 2020).

[2] Lenz HJ, Lee FC, Haller DG, et al. Extended safety and efficacy data on S-1 plus cisplatin in patients with untreated, advanced gastric carcinoma in a multicenter phase II study. Cancer 2007; 9: 33-40. https://doi.org/10.1002/cncr.22329

[3] Koizumi W, Narahara H, Hara T, et al. S-1 plus cisplatin versus S-1 alone for first line treatment of advanced gastric cancer (SPIRITS trial): A phase III trial. Lancet Oncol 2008; 9: $215-221$ https://doi.org/10.1016/S1470-2045(08)70035-4

[4] Hironaka S, Sugimoto N, Yamaguchi K, et al. S-1 plus cisplatin leucovorin versus $\mathrm{S}-1$ plus leucovorin and oxaliplatin versus $\mathrm{S}-1$ plus cisplatin in patients with advanced gastric cancer: a randomized, multicenter, open-label, phase 2 trial. Lancet Oncol 2016; 17: 99-108.

https://doi.org/10.1016/S1470-2045(15)00410-6

[5] Ina K, Hirade K, Kabeya $\mathrm{M}$, et al. Long-term survivors of metastatic gastric cancer for $>5$ years after chemotherapy initiation. Cancer Reports and Reviews 2019; 3: 1-5. https://doi.org/10.15761/CRR.1000183

[6] Chen DS, Mellman I. Oncology meets immunology: the cancer-immunity. Immunity 2013; 39: 1-10. https://doi.org/10.1016/j.immuni.2013.07.012

[7] Topalian SL, Drake CG, Pardoll DM. Targeting the PD-1/ B7H1 (PD-L1) pathway to activate anti-tumor immunity. Curr Opin Immunol 2012; 24: 207-2012. https://doi.org/10.1016/j.coi.2011.12.009

[8] Pardoll DM. The blockade of immune checkpoints in cancer immunotherapy. Nat Rev Cancer 2012; 12: 252-264. https://doi.org/10.1038/nrc3239

[9] Taube JM, Klein A, Brahmer JR, et al. Association of PD-1, PD-1 ligands, and other features of the tumor immune 
microenvironment with response to anti-PD-1 therapy. Clin Cancer Res 2014; 20: 5064-5074.

https://doi.org/10.1158/1078-0432.CCR-13-3271

[10] Thompson ED, Zahurk M, Murphy A, et al. Patterns of PD-L1 expression and CD8 $\mathrm{T}$ cell infiltration in gastric adenocarcinomas and associated immune stroma. Gut 2016. https://doi.org/10.1136/gutjnl-2015-310839

[11] Tumeh PC, Harview CL, Yearly JH, et al. PD-L1 blockade induces responses by inhibiting adaptive immune resistance. Nature 2014; 515: 568-571.

https://doi.org/10.1038/nature13954

[12] Concha-Benavente F, Srivastava RM, Trivedi S, et al. Identification of the Cell-Intrinsic and -Extrinsic Pathways Downstream of EGFR and IFNy That Induce PD-L1 Expression in Head and Neck Cancer. Cancer Res 2016; 76: 1031-43.

https://doi.org/10.1158/0008-5472.CAN-15-2001

[13] Jiang X, Zhou J, Giobbie-Hurder A, Wargo J, Hodi FS. The association of MAPK in melanoma cells resistant to BRAF inhibition promotes PD-L1 expression that is reversible by MEK and PI3K inhibition. Clin Cancer Res 2013 19; 598-609. https://doi.org/10.1158/1078-0432.CCR-12-2731

[14] Stutvoet T, Kol A, de Vries EG, et al. MAPK pathway activity plays a key role. J Pathol 2019; 249: 52-64. https://doi.org/10.1002/path.5280

[15] Parsa AT, Waldron JS, Panner A, et al. Loss of tumor suppressor PTEN function increases B7-H1 expression and immunoresistance in glioma. Nat Med 2007; 13: 84-88. https://doi.org/10.1038/nm1517

[16] Wolfle SJ, Strebovsky J, Bartz H, et al. PD-L1 expression on tolerogenic APCs is controlled by STAT-3. Eur J Immunol 2011; 41: 413-424. https://doi.org/10.1002/eji.201040979

[17] Bouillez A, Rajabi H, Jin C, et al. MUC-1 integrates PD-L1 induction with repression of immune effectors in non-smallcell lung cancer. Oncogene 2017; 36: 4037-46. https://doi.org/10.1038/onc.2017.47

[18] Latchman Y, Wood CR, Chernova T, et al. PD-L2 is a second ligand for $\mathrm{PD}-1$ and inhibits $\mathrm{T}$ cell activation. Nat Immunol 2001; 2: 261-8.

https://doi.org/10.1038/85330

[19] Schmid P, Hegde PS, Zou W, et al. Association of PD-L2 expression in human tumors with atezolizumab activity. J Clin Oncol 2016; 34(Suppl 15): 11506.

https://doi.org/10.1200/JCO.2016.34.15 suppl.11506

[20] Yearly JH, Gibson C, Yu N, et al. PD-L2 expression in human tumors: Relevance to anti-PD-1 therapy in cancer. Clin Cancer Res 2017; 23: 3158-67. https://doi.org/10.1158/1078-0432.CCR-16-1761

[21] Chinara G, Hamuro J, Maeda Y, Arai Y, Fukuoka F. Fractionation and purification of the polysaccharides with marked antitumor activity, especially lentinan, from Lentinus edodes (Berk.) Sing Cancer Res 1970; 30: 2776-2781.

[22] Ina K, Kataoka T, Ando T. The use of lentinan for treating gastric cancer. Anticancer Agents Med Chem 2013; 13: 6818.

https://doi.org/10.2174/1871520611313050002

[23] Oba K, Kobayashi M, Matsui T, Kodera Y, Sakamoto J. Individual patient based meta-analysis of lentinan for unresectable/recurrent gastric cancer. Anticancer Res 2009; 29: 2739-46.

[24] Wang H, Cai Y, Zheng Y, Bai Q, Xie D, Yu J. Efficacy of biological response modifier lentinan with chemotherapy for advanced cancer: a meta-analysis. Cancer Med 2017; 6: 2222-33.

https://doi.org/10.1002/cam4.1156

[25] Ren L, Perera C, Hemar Y. Antitumor activity of mushroom polysaccharides: a review. Food Funct 2012; 3: 1118-30. https://doi.org/10.1039/c2fo10279j
[26] Aleem, E. $\beta$-glucans and their applications in cancer therapy: focus on human studies. Anticancer Agents Med Chem 2013; 13: 709-19.

https://doi.org/10.2174/1871520611313050005

[27] Yoshino S, Nishikawa K, Morita S, et al. Randomised phase III study of S-1 alone versus S-1 plus lentinan for unresectable or recurrent gastric cancer. Eur J Cancer 2016; 65: 164-71.

https://doi.org/10.1016/j.ejca.2016.06.012

[28] Higashi D, Seki K, Ishibashi $Y$, et al. The effect of lentinan combination therapy for unresectable advanced gastric cancer. Anticancer Res 2012; 32: 2365-8.

[29] Ina $\mathrm{H}$, Yoneda $\mathrm{M}$, Kanda $\mathrm{M}$, et al. Lentinan, a shiitake mushroom $\beta$-glucan, stimulates tumor-specific adaptive immunity through PD-L1 down-regulation in gastric cancer cells. Med Chem (Los Angeles) 2016; 6: 710-4.

https://doi.org/10.4172/2161-0444.1000419

[30] Schefe JH, Lehmann KE, Buchsmann IR, Unger T, FunkeKaiser H. Quantitative real-time RT-PCR data analysis: current concepts and the novel gene expression's CT difference formula. J Mol Med 2006; 84: 901-10.

https://doi.org/10.1007/s00109-006-0097-6

[31] Inoue $\mathrm{Y}$, Yoneda $\mathrm{M}$, Zhao J, et al. Molecular cloning and characterization of chick SPACRCAN. J Biol Chem 2006; 281: 10381-8.

https://doi.org/10.1074/jbc.M508161200

[32] Yamada $\mathrm{H}$, Yoneda $\mathrm{M}$, Inaguma $\mathrm{S}$, et al. Infliximab counteracts tumor necrosis factor- $\alpha$-enhanced induction of matrix metalloproteinases that degrade claudin and occludin in non-pigmented ciliary epithelium. Biochem Pharmacol 2013; 85: 1770-82

https://doi.org/10.1016/j.bcp.2013.04.006

[33] Kanda $Y$. Investigation of the freely-available easy-to-use software "EZR" (Easy R) for medical statistics. Bone Marrow Transplant 2013; 48: 452-8.

https://doi.org/10.1038/bmt.2012.244

[34] Grabosch S, Bulatovic M, Zeng F, et al. Cisplatin-induced immune modulation in ovarian cancer mouse models with distinct inflammation profiles. Oncogene 2018; 38: 2380-93. https://doi.org/10.1038/s41388-018-0581-9

[35] Fournel L, Wu Z, Stadler N, et al. Cisplatin increases PD-L1 expression and optimizes immune check-point blockade in non-small lung cancer. Cancer Letters 2019; 464: 5-14. https://doi.org/10.1016/j.canlet.2019.08.005

[36] Paz-Ares L, Luft A, Vicente $\mathrm{D}$, et al. Pembrolizumab plus chemotherapy for squamous non-small-cell lung cancer. $\mathrm{N}$ Engl J Med 2018; 379; 2040-51. https://doi.org/10.1056/NEJMoa1810865

[37] Gadgeel S, Rodoriguez-Abreu D, Speranza G, et al. Updated analysis from KEYNOTE-189: Pembrolizumab or placebo plus pemetrexed an previously untreated metastatic nonsquamous non-small cell lung cancer. J Clin Oncol 2020; JCO1903136. https://doi.org/10.1200/JCO.19.03136

[38] Reck M, Rodríguez-Abreu $D$, Robinson AG, et al Pembrolizumab versus chemotherapy for PD-L1-positive non-small-cell lung cancer. N Engl J Med 2016; 375: 182333.

https://doi.org/10.1056/NEJMoa1606774

[39] Chen J, Jiang CC, Jin L, Zhang XD. Regulation of PD-L1: A novel role of pro-survival signaling in cancer. Ann Oncol 2016; 27: 409-16. https://doi.org/10.1093/annonc/mdv615

[40] Sun C, Mezzadra R, Schumacher T. Regulation and function of the PD-L1 checkpoint. Immunity 2018; 48: 434-52. https://doi.org/10.1016/j.immuni.2018.03.014

[41] Gowrishanker K, Gunatilake D, Gallagher SJ, Tiffen J, Rizos $\mathrm{H}$, Hersey P. Inducible but not constitutive expression of 
PD-L1 in human melanoma cells is dependent on activation of NF- $\kappa \beta$. PLoS One 2015; 10.

https://doi.org/10.1371/journal.pone.0123410

[42] Vetvicka V, Vetvickova J. Anti-infectious and anti-tumor activities of $\beta$-glucans. Anticancer Res 2020; 40: 3139-45. https://doi.org/10.21873/anticanres.14295

[43] Xu X, Pan C, Zhang L, Ashida H. Immunomodulatory $\beta$ glucan from Lentinus edodes activates mitogen-activated protein kinases and nuclear factor- $\kappa \beta$ in murine RAW 264.7 macrophages. J Biol Chem 2011; 286: 31194-8. https://doi.org/10.1074/jbc.M111.246470
[44] Chihara G, Maeda Y, Hamuro J, et al. Inhibition of mouse sarcoma 180 by polysaccharides from Lentinus edodes (Berk.) Sing. Nature 1969; 222: 687-8. https://doi.org/10.1038/222687a0

[45] Ina K, Furuta R. Image of Month: Complete response of metastatic gastric cancer to chemo-immunotherapy. Indian J Med Res 2017; 146: 141.

https://doi.org/10.4103/ijmr.IJMR 13216

Received on 25-06-2020

Accepted on 16-07-2020

Published on 31-07-2020

https://doi.org/10.30683/1927-7229.2020.09.01

(C) 2020 Ina et al.; Licensee Neoplasia Research.

This is an open access article licensed under the terms of the Creative Commons Attribution Non-Commercial License (http://creativecommons.org/licenses/by-nc/3.0/) which permits unrestricted, non-commercial use, distribution and reproduction in any medium, provided the work is properly cited. 